\title{
Plasma instabilities in high electric fields
}

\author{
Morawetz, K.; Jauho, Antti-Pekka
}

Published in:

Physical Review E. Statistical, Nonlinear, and Soft Matter Physics

Link to article, DOI:

10.1103/PhysRevE.50.474

Publication date:

1994

Document Version

Publisher's PDF, also known as Version of record

Link back to DTU Orbit

Citation (APA):

Morawetz, K., \& Jauho, A-P. (1994). Plasma instabilities in high electric fields. Physical Review E. Statistical, Nonlinear, and Soft Matter Physics, 50(1), 474-479. https://doi.org/10.1103/PhysRevE.50.474

\section{General rights}

Copyright and moral rights for the publications made accessible in the public portal are retained by the authors and/or other copyright owners and it is a condition of accessing publications that users recognise and abide by the legal requirements associated with these rights.

- Users may download and print one copy of any publication from the public portal for the purpose of private study or research.

- You may not further distribute the material or use it for any profit-making activity or commercial gain

- You may freely distribute the URL identifying the publication in the public portal

If you believe that this document breaches copyright please contact us providing details, and we will remove access to the work immediately and investigate your claim. 


\title{
Plasma instabilities in high electric fields
}

\author{
K. Morawetz \\ Max-Planck-Gesellschaft, AG "Theoretische Vielteilchenphysik" an der Universität Rostock, 18051 Rostock, Germany \\ A.P. Jauho \\ Mikroelektronik Centret, Technical University of Denmark, DK-2800 Lyngby, Denmark \\ and Nordisk Institut for Teoretisk Fysik, Blegdamsvej 17, DK-2100 Copenhagen Ø, Denmark
}

(Received 24 June 1993; revised manuscript received 11 March 1994)

\begin{abstract}
We analyze nonequilibrium screening with nonequilibrium Green function techniques. By employing the generalized Kadanoff-Baym ansatz to relate the correlation function to the nonequilibrium distribution function, the latter of which is assumed to be a shifted Maxwellian, an analytically tractable expression is derived for the nonequilibrium dielectric function $\epsilon(\mathbf{K}, \omega)$. For certain values of momenta $\mathbf{K}$ and frequency $\omega, \operatorname{Im} \epsilon(K, \omega)$ becomes negative, implying a plasma instability. This new instability exists only for strong electric fields, underlining its nonequilibrium origin.
\end{abstract}

PACS number(s): $52.35 . \mathrm{Qz}, 52.25 . \mathrm{Mq}, 72.20 . \mathrm{Ht}, 72.15 . \mathrm{Nj}$

\section{INTRODUCTION}

Considerable interest has been devoted to instabilities in plasmas [1-4]. Plasma systems are characterized by long range Coulomb forces, which result in collective phenomena such as plasma oscillations. In the present paper we present a quantum statistical investigation of plasmas under the influence of strong electric fields. In the recent past a number of attempts have been made to study screening in nonequilibrium semiconductor systems, e.g., by Lowe and Barker [5]. $\mathrm{Hu}$ and coworkers $[6,7]$ compared the linear dielectric function derived either from the semiclassical Boltzmann equation, or from the quantum transport equation of Baym and Kadanoff. It was found that in the long wavelength limit $1 / q \gg \lambda_{B}\left(\lambda_{B}\right.$ is the de Broglie wavelength) the semiclassical and quantum results coincide. Further, $\mathrm{Hu}$ and coworkers [6,7] showed that the Kadanoff-Baym quantum transport equation leads to a dielectric function, which has the Lindhard form, but where the Fermi distributions are replaced by nonequilibrium distribution functions. The nonlinear dielectric functions, which we derive in this work, reproduce this result in the limit of weak fields. Further, $\mathrm{Hu}$ and O'Connell [8] have generalized the Lindhard dielectric function to include the effect of electron density fluctuations. The work described in [6-8] is essentially limited to linear response. Arbitrarily strong uniform fields have been treated by employing the Airy-transform technique [9-11]. In particular, Yi, Kriman, and Ferry [12] found a descreening effect in high applied electric fields. The final formal result of Ref. [12] involves a nonequilibrium distribution function, which should be determined from a quantum transport equation, and hence no detailed numerical results for the dielectric function were presented. In our work we follow an alternative route: by applying the generalized Kadanoff-Baym ansatz [13] to relate the nonequilibrium correlation function to the momentum distribution function, for which we use a physical model, we are able to extract an explicit analytic result for the nonequilibrium dielectric function. We also present numerical results, and find a remarkable phenomenon: the imaginary part of the dielectric function becomes negative for a range of electric fields and frequencies, thus pointing towards the existence of a new unstable region.

\section{NONEQUILIBRIUM DIELECTRIC FUNCTION}

We consider a system of fermions (charge $Z_{i} e$ ) under the influence of an applied constant electric field. Using the vector potential gauge we have the following Hamiltonian:

$$
\begin{aligned}
H= & \sum_{i} \int d \mathbf{r} \Psi_{i}^{\dagger}(\mathbf{r}, t) H_{i}\left(\frac{\hbar}{i} \nabla-\frac{Z_{i} e}{c} \mathbf{A}(t)\right) \Psi_{i}(\mathbf{r}, t) \\
& +\frac{1}{2} \sum_{i, j} \int d \overline{\mathbf{r}} d \mathbf{r} \Psi_{i}^{\dagger}(\mathbf{r}, t) \Psi_{j}^{\dagger}(\overline{\mathbf{r}}, t) V_{i j}(\mathbf{r}-\overline{\mathbf{r}}) \\
& \times \Psi_{i}(\mathbf{r}, t) \Psi_{j}(\overline{\mathbf{r}}, t)
\end{aligned}
$$

where $H_{i}$ is the one-body Hamiltonian, and

$$
V_{i j}(\mathbf{r}-\overline{\mathbf{r}})=\frac{e^{2} Z_{i} Z_{j}}{|\mathbf{r}-\overline{\mathbf{r}}|}
$$

To examine the nonequilibrium properties of this system we introduce the correlation functions

$$
\begin{gathered}
g^{>}\left(\mathbf{r}, t, \mathbf{r}^{\prime}, t^{\prime}\right)=-i\left\langle\Psi(\mathbf{r}, t) \Psi^{\dagger}\left(\mathbf{r}^{\prime}, t^{\prime}\right)\right\rangle \\
g^{<}\left(\mathbf{r}, t, \mathbf{r}^{\prime}, t^{\prime}\right)=i\left\langle\Psi^{\dagger}\left(\mathbf{r}^{\prime}, t^{\prime}\right) \Psi(\mathbf{r}, t)\right\rangle .
\end{gathered}
$$

It is often advantageous to use the center-of-mass and difference coordinates, and express the correlation functions $g^{<,>}$in terms of the nonequilibrium spectral function $a(\mathbf{p}, \omega, \mathbf{R}, T)$ (recall that $\left.g^{<}-g^{>} \equiv i a\right)$ : 


$$
\begin{gathered}
g^{<}(\mathbf{p}, \omega, \mathbf{R}, T)=i a(\mathbf{p}, \omega, \mathbf{R}, T) F(\mathbf{p}, \omega, \mathbf{R} . T) \\
g^{>}(\mathbf{p}, \omega, \mathbf{R}, T)=-i a(\mathbf{p}, \omega, \mathbf{R}, T)[1-F(\mathbf{p}, \omega, \mathbf{R}, T)],
\end{gathered}
$$

where $F(\mathbf{p}, \omega, \mathbf{R}, T)$ is a "quantum distribution function" [21], which should be determined by solving the appropriate quantum transport equation. If the quasiparticle picture is valid, i.e., the spectral function is a sharply peaked function with a maximum at the quasiparticle energy, one can simplify Eq. (4) to

$$
g^{<}(\mathbf{p}, \omega, \mathbf{R}, T)=i a(\mathbf{p}, \omega, \mathbf{R}, T) f_{W}(\mathbf{p}, \mathbf{R}, T)
$$

where $f_{W}$ is the Wigner distribution function. We refer to this relation as the Kadanoff-Baym ansatz.

Consider now the spectral function of free particles within a static and uniform external electric field [14]:

$$
A_{0}(\mathbf{K}, \omega, \mathbf{R}, T)=\frac{2 \pi}{\lambda_{E}} \mathrm{Ai}\left[\frac{1}{\lambda_{E}}\left(\hbar \omega-\frac{K^{2}}{2 m}\right)\right],
$$

where $\operatorname{Ai}(x)$ is the Airy function, $\mathbf{K}=\mathbf{p}+e \mathbf{E T}$ is the kinematical momentum, and $\lambda_{E}$ is given by

$$
\lambda_{E}^{3}=\frac{\hbar^{2} e^{2} E^{2}}{8 m} \text {. }
$$

Since $A_{0}(\mathbf{K}, \omega, \mathbf{R}, T)$ has no single dominant peak, the simple replacement Eq. (5) has no a priori justification.

Lipavsky et al. [13] have given an alternative ansatz for relating the correlation function and the distribution function, which, in fact, is exact for the Hartree-Fock approximation for the self-energy. For a parabolic dispersion law and constant fields it reads [15]

$$
\begin{aligned}
g^{<}(\mathbf{K}, \tau, \mathbf{R}, T)= & i \exp \left[-\frac{i}{\hbar}\left(\epsilon_{K} \tau+\frac{e^{2} E^{2}}{24 m} \tau^{3}\right)\right] \\
& \times f_{W}\left(\mathbf{K}-\frac{e \mathbf{E}|\tau|}{2}\right) .
\end{aligned}
$$

Note that in Eq. (8) one uses the variable $\tau$ rather than its Fourier transform $\omega$ [see Eqs. (5) and (4)]. This ansatz is superior to the Kadanoff-Baym ansatz in the case of high external fields in several respects: (i) it has the correct spectral properties, (ii) it is gauge invariant, (iii) it preserves causality, (iv) the quantum kinetic equations derived with Eq. (8) coincide with those obtained with the density matrix technique [16], and (v) it reproduces the Debye-Onsager relaxation effect [17]. The retardation in the momentum variable of the distribution function in Eq. (8) turns out to be essential for the results found below.

In the random phase approximation (RPA) the dielectric function has the following structure:

$$
\begin{aligned}
\epsilon(\mathbf{K}, \tau)= & \delta(\tau)+i \Theta(\tau) V(\mathbf{K}) \int \frac{d \overline{\mathbf{p}}}{(2 \pi \hbar)^{3}}\left[g^{>}(\overline{\mathbf{p}}, \tau)\right. \\
& \left.\times g^{<}(\overline{\mathbf{p}}-\mathbf{K},-\tau)-g^{<}(\overline{\mathbf{p}}, \tau) g^{>}(\overline{\mathbf{p}}-\mathbf{K},-\tau)\right]
\end{aligned}
$$

Using the ansatz (8) and Fourier transforming we find

$$
\begin{aligned}
\epsilon(\mathbf{K}, \omega, \mathbf{R}, T)= & +V(\mathbf{K}) \int \frac{d \overline{\mathbf{p}}}{(2 \pi \hbar)^{3}} \Psi(\omega, \mathbf{K}, \overline{\mathbf{p}}) \\
& \times[f(\overline{\mathbf{p}}-\mathbf{K})-f(\overline{\mathbf{p}})]
\end{aligned}
$$

where the two particle spectral function is given by

$$
\Psi(\omega, \mathbf{K}, \overline{\mathbf{p}})=\int \frac{d \tau d \bar{\omega}}{2 \pi} \frac{\exp \left(i \bar{\omega} \tau-i \frac{e \mathbf{E} \cdot \mathbf{K} \tau^{2}}{2 m \hbar}\right)}{\epsilon_{\overline{\mathbf{p}}}-\epsilon_{\overline{\mathbf{p}}-\mathbf{K}}+\hbar(\bar{\omega}-\omega)-i \eta}
$$

If we neglect the E-dependent term in the exponential, and use equilibrium distribution functions in (10), we recover the usual Lindhard result.

\section{ANALYTICAL EXPRESSIONS}

In (non)degenerate systems electron-electron scattering tends to drive the nonequilibrium distribution towards a heated displaced Fermi-Dirac (Maxwellian) distribution function [18]. Further, we assume that some inelastic scattering process stabilizes the system so that a stationary state can be reached $[19,20]$. Thus, rather than solving the quantum kinetic equation (with the appropriate scattering mechanisms) for the nonequilibrium distribution function, a physically motivated approach is to evaluate (10) with a displaced Maxwellian distribution:

$$
f_{W}(\mathbf{p})=n \lambda^{3}\left(T_{E}\right) \exp \left[-\frac{(\mathbf{p}-\mathbf{Q})^{2}}{2 m k_{B} T_{E}}\right]
$$

where $\lambda^{2}=\frac{(2 \pi \hbar)^{2}}{m k_{B} T_{E}}$. The electron temperature $T_{E}$ and drift momentum $\mathbf{Q}(\mathbf{E})$ are field dependent, and should, in principle, be evaluated self-consistently from the kinetic equation. This is, however, not necessary for our present purposes. The great advantage of this model distribution function is that the integrals in (10) can be performed analytically. Using contour integration techniques [22], one finds

$$
\begin{aligned}
\epsilon(\mathbf{K}, \omega)= & 1-\frac{\kappa^{2} k_{B} T_{E} \hbar^{2} \sqrt{\pi}}{K^{3} v_{T}} i \sqrt{\beta} \\
& \times\left\{w\left[\left(\frac{K}{2 m v_{T}}+\frac{m \omega \hbar-\mathbf{Q} \cdot \mathbf{K}}{m K v_{T}}\right) \sqrt{\beta}\right]\right. \\
& \left.-w\left[\left(\frac{-K}{2 m v_{T}}+\frac{m \omega \hbar-\mathbf{Q} \cdot \mathbf{K}}{m K v_{T}}\right) \sqrt{\beta}\right]\right\}
\end{aligned}
$$

with

$$
\sqrt{\beta}=\frac{1}{\sqrt{1+i a}}=\frac{\cos \left(\frac{\phi}{2}\right)-i \sin \left(\frac{\phi}{2}\right)}{\left(1+16 a^{2}\right)^{1 / 4}} .
$$

Here $\tan \phi=a$, and we have introduced the dimensionless field parameter $a=\frac{e E \cdot K}{\sqrt{\pi}} K^{2} \frac{\lambda\left(T_{E}\right)}{v_{T}}$, and used the thermal velocity $v_{T}=\sqrt{\frac{2 k_{B} T_{E}}{m}}$. The inverse Debye screening
length $\kappa$ is given by

$$
\kappa^{2}=\frac{4 \pi e^{2} Z^{2} n}{k_{B} T_{E}}
$$


Finally, $w(x)$ reads

$$
w(x)=e^{-x^{2}} \operatorname{erfc}(-i x),
$$

where erfc stands for the complement of the error function [23]. These results can be generalized to the degenerate case by using a shifted Fermi distribution instead of (12). The final result can be expressed in terms of the nondegenerate dielectric function $[\epsilon(\sqrt{\beta})-1]$ from Eq. (13):

$$
(\epsilon-1)_{\mathrm{deg}}=\frac{1}{n \lambda^{3}} \sum_{\nu=0}^{\infty} \frac{(-1)^{\nu} z^{\nu+1}}{(\nu+1)^{3 / 2}}\left[\epsilon\left(\sqrt{\beta_{\nu}}\right)-1\right],
$$

where $z=e^{\mu /\left(k_{B} T_{E}\right)}$ and $\sqrt{\beta_{\nu}}=\left(\frac{1}{\nu+1}+i a\right)^{-1 / 2}$.

Before turning to a detailed numerical analysis, it is instructive to consider two special cases. First, in the limit of vanishing fields (which also coincides with the case of wave propagation perpendicular to the field), the constant $a$ vanishes, and one finds

$$
\begin{aligned}
\epsilon(\mathbf{K}, \omega)= & 1+\frac{\kappa^{2} k_{B} T_{E} \hbar^{2} \sqrt{\pi}}{K^{3} v_{T}} \\
& \times i\left[w\left(\frac{K}{2 m v_{T}}+\frac{m \omega \hbar-\mathbf{Q} \cdot \mathbf{K}}{m K v_{T}}\right)\right. \\
& \left.-w\left(-\frac{K}{2 m v_{T}}+\frac{m \omega \hbar-\mathbf{Q} \cdot \mathbf{K}}{m K v_{T}}\right)\right] .
\end{aligned}
$$

This is the well-known result for the quantum RPA dielectric function for Maxwellian plasmas and can be rewritten in terms of ${ }_{1} F_{1}$ functions [24].

The parameter $a$, which is nonvanishing for longitudinal fields, depends on the de Broglie wavelength, and we infer that the results found in this work are a consequence of quantum interference.

Second, the classical limit is recovered if one expands Eq. (13) in powers of $\hbar$. Using $\mathbf{K}=\hbar \mathbf{k}$, where $\mathbf{k}$ is the wave vector, one finds

$$
\begin{aligned}
\epsilon(\mathbf{k}, \omega)= & +\frac{\kappa^{2}}{k^{2}} \beta_{\mathrm{cl}}\left[1+i \frac{m \omega-\mathbf{Q} \cdot \mathbf{k}}{m k v_{T}} \sqrt{\pi} \sqrt{\beta_{\mathrm{cl}}}\right. \\
& \left.\times w\left(\frac{m \omega-\mathbf{Q} \cdot \mathbf{k}}{m k v_{T}} \sqrt{\beta_{\mathrm{cl}}}\right)\right] .
\end{aligned}
$$

$\beta_{\mathrm{cl}}$ is given by (14) with the classical limit of the parameter $a=\frac{e \mathbf{E} \cdot \mathbf{k}}{k^{2} k_{B} T}$. This result for the field free $(\sqrt{\beta}=1)$ dielectric function has been extensively discussed in the literature [1].

\section{NUMERICAL RESULTS}

We now turn to the analysis of the central result for $\epsilon(\mathbf{K}, \omega)$, Eq. (13). In Fig. 1 we have plotted the real part of Eq. (13) as a function of the applied electric field strength. It can be seen that the zeros of $\operatorname{Re}[\epsilon(\mathbf{K}, \omega)]$ and the value of $\omega$ at which $\operatorname{Re}[\epsilon(K, \omega)]$ reaches a minimum move to higher values of $\omega$ with increasing fields. This indicates that the plasma frequency is enhanced by the applied electric field. The lower zero corresponds to

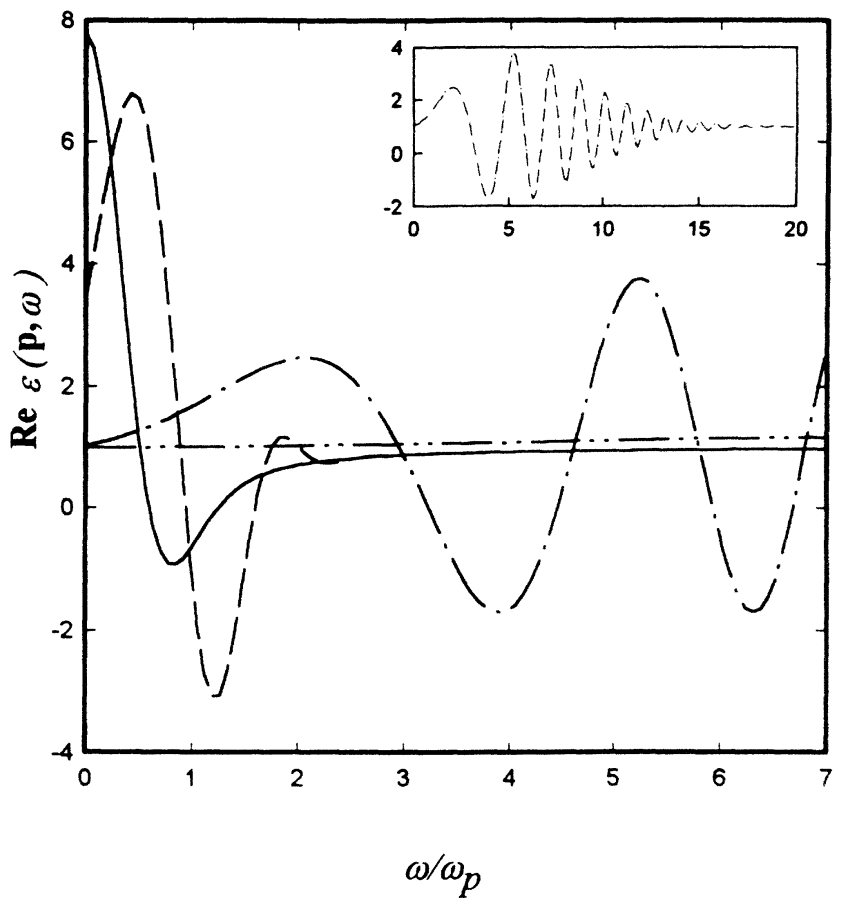

FIG. 1. The real part of quantum RPA dielectric function vs frequency for wave vector $k=0.00384 a_{0}^{-1}$. The assumed temperature is $T=16000 \mathrm{~K}$ and the Debye screening length $100 a_{0}$. The deviation from the field free curve (solid line) is plotted for three different field strengths: $E=10^{8} \mathrm{~V} / \mathrm{m}$ (dashed line), $E=10^{9} \mathrm{~V} / \mathrm{m}$ (dash-dotted line), and $E=10^{10}$ $\mathrm{V} / \mathrm{m}$ (dash-double-dotted line). The case $E=10^{9} \mathrm{~V} / \mathrm{m}$ is shown as an inset in order to illustrate the oscillating behavior at special field strengths.

an acoustic excitation. This is normally connected with a large imaginary part of the dielectric function, and therefore it can be considered as a quasiparticle excitation. For very high field strengths the screening vanishes, which is in accordance with the descreening effect found in [12].

In Fig. 2 we display the imaginary part of the dielectric function. There a similar behavior occurs: the maximum of damping moves to higher values of $\omega$ for increasing fields. It is noteworthy that $\operatorname{Im}[\epsilon(\mathbf{K}, \omega)]$ becomes negative at certain frequencies and fields. This behavior suggests the existence of unstable modes. Recall that the mean field energy $Q$ gained from (or lost to) a longitudinal test wave with amplitude $E_{1}$ in the plasma can be expressed as [25]

$$
Q=\frac{\varepsilon_{o}}{2} \omega\left|\mathbf{E}_{\mathbf{1}}\right|^{2} \operatorname{Im}[\epsilon(\mathbf{K}, \omega)] .
$$

Thus, a change of the sign in the imaginary part of the dielectric function indicates a change in the direction of energy transfer. This can be interpreted as a propagating wave gaining more energy from the surrounding plasma than losing to it, thus being unstable.

This interpretation is further confirmed by studying the excitation spectrum $\operatorname{Im} \epsilon^{-1}(\mathbf{K}, \omega)$, which is plotted in Fig. 3. In addition to the shift of ordinary plasmon exci- 


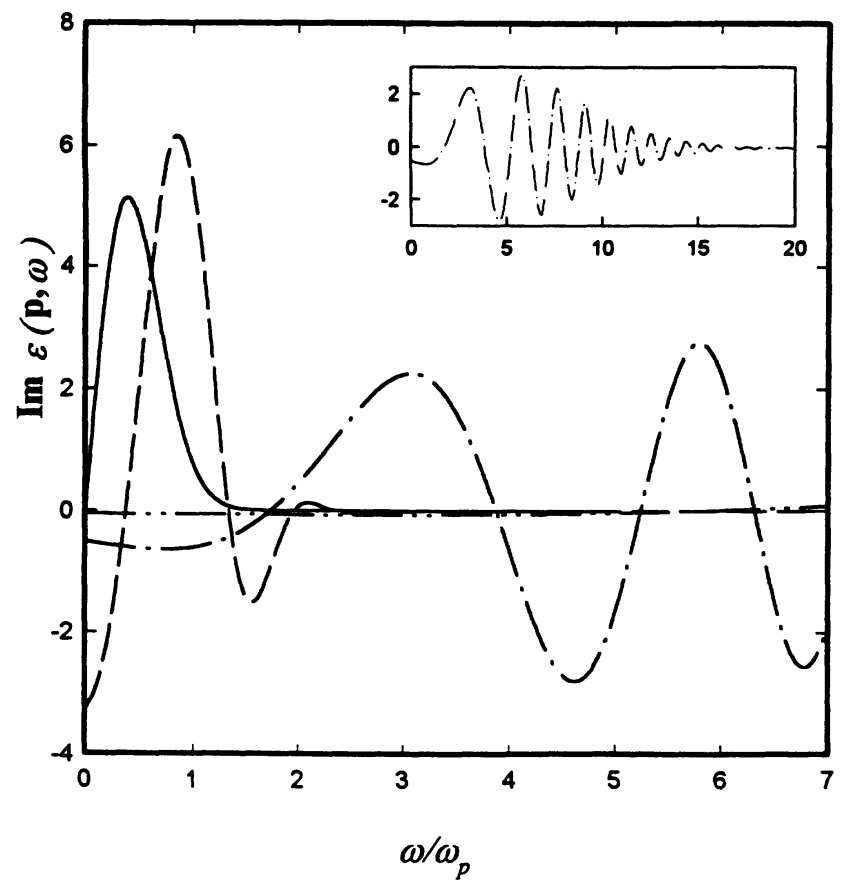

FIG. 2. The imaginary part of quantum RPA dielectric function vs frequency. The parameters are as in Fig. 1.

tations to higher values of $\omega$, we find that for frequencies higher than the plasmon frequency a negative excitation occurs. This is an unstable mode. For field strengths exceeding $10^{9} \mathrm{~V} / \mathrm{m}$ no excitations were found due to the descreening effect described above.

The instability found in our work has another origin

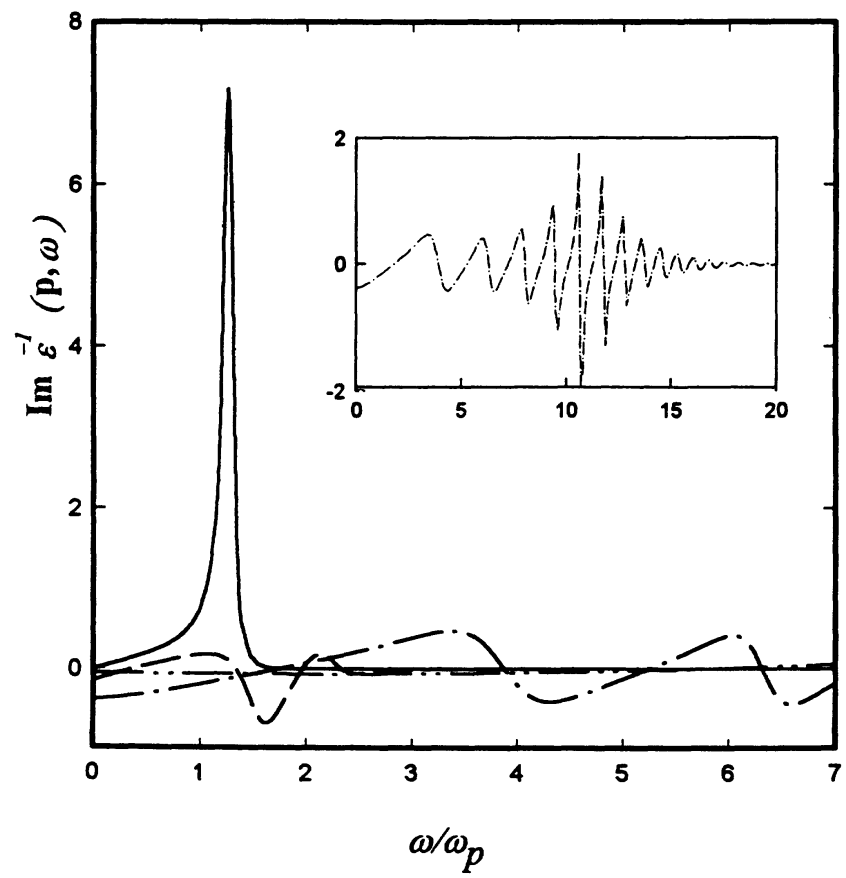

FIG. 3. The spectral function of the excitation spectrum $\operatorname{Im} \epsilon^{-1}$ of quantum RPA dielectric function vs frequency. The parameters are as in Fig. 1. than the ones discussed conventionally [1]. Normally one would attempt to calculate the influence of the applied electric field on the distribution function and, with the help of the Penrose [26] criterion, search for regions where an instability may occur [27].

In order to obtain more physical insight into the nature of the instability we consider the long wavelength limit of the classical expression (19):

$$
\epsilon(\mathbf{k}, \omega)=1-\frac{\omega_{p}^{2}}{\omega^{2}}\left[1+6 i \frac{e \mathbf{E} \cdot \mathbf{k}}{m \omega^{2}}\right]+O\left(k^{2}\right) .
$$

The energy gain (20) takes the form

$$
Q=-3 \varepsilon_{0} E_{1}^{2} \frac{e \mathbf{E} \cdot \mathbf{k} \omega_{p}^{2}}{m \omega^{3}}+O\left(k^{3}\right),
$$

where the negative sign indicates the growth of the wave. This instability can be understood as a nonlinear Landau damping for small $k$ in high electric fields. This is confirmed by the fact that the energy transfer (22) is completely independent of collisions. In the Appendix we give an alternative derivation of this nonlinear Landau damping in high fields.

The instability discovered in the present paper follows from using the generalized Kadanoff-Baym (GKB) ansatz, which is an exact relation in the Hartree-Fock approximation of the self-energy. Had we used the older $\mathrm{KB}$ ansatz [5], no instability would have occurred. This underlines the care required when working with nonequilibrium Green functions.

Our findings for the dielectric function (DF) can be summarized as follows.

(i) The acoustic mode, which corresponds to the lower zero of the real part of the DF, tends to higher values of $\omega$ with increasing fields. The damping represented by the imaginary part of the DF is diminished in this region due to the field. These properties of the DF mean that the acoustic mode starts to become free, i.e., it is not damped further to quasiparticle excitations and can now be observed [27].

(ii) For certain electric fields and frequencies the imaginary part of the DF changes its sign. This is reflected in negative excitations in the spectral function. By considering the mean field energy, it is found that in this case the energy transfer from a test wave to the plasma changes direction, and an instability occurs.

In a forthcoming paper we will present calculations for a partially ionized hydrogen plasma also including the influence of the applied electric field on the distribution function [28].

\section{APPENDIX: PHYSICAL DERIVATION OF NONLINEAR LANDAU DAMPING IN HIGH ELECTRIC FIELDS}

In order to derive the nonlinear Landau damping in high electric fields we divide the distribution function $f_{0}(u)$ of the plasma up into beams of velocity $u$ with density $n_{u}$ and examine their motion in a wave $E_{1} \sin (k x-\omega t)$ and an external field $E$. Our derivation 
follows closely the analysis of Chen [29].

The linearized fluid equation of beams with the velocity disturbance $v_{1}$ becomes in one dimension

$$
m\left(\frac{\partial v_{1}}{\partial t}+u \frac{\partial v_{1}}{\partial x}\right)=-e E_{1} \sin (k x-\omega t)-e E .
$$

Now we seek for a solution which ensures the initial conditions $v_{1}=0$ at $t=0$. This is necessary, because otherwise $v_{1}$ may become very large in the vicinity of $u=\omega / k$ and the plasma would be in a specially prepared state initially. The solution reads

$v_{1}=-\frac{e E_{1}}{m} \frac{\cos (k x-w t)-\cos (k x-k u t)}{\omega-k u}-\frac{e E}{m} t$.

The corresponding continuity equation for the density disturbance $n_{1}$ is

$$
\frac{\partial n_{1}}{\partial t}+u \frac{\partial n_{1}}{\partial x}=-n_{u} \frac{\partial v_{1}}{\partial x}
$$

Imposing the same boundary conditions $\left(n_{1}=0\right.$ at $\left.t=0\right)$ the solution is

$$
n_{1}=-n_{u} \frac{e E_{1} k}{m} \frac{\cos (k x-\omega)-\cos (k x-k u t)-(\omega-k u) t \sin (k x-k u t)}{(\omega-k u)^{2}}
$$

The work done by the wave on each beam is calculated as follows. The force acting on unit volume of each beam is

$$
F_{u}=\left[-e E_{1} \sin (k x-\omega t)-e E\right]\left(n_{u}+n_{1}\right)
$$

The energy changes at the rate

$$
\frac{d W_{u}}{d t}=F_{u}\left(u+v_{1}\right)
$$

We next perform a spatial average, and sum over all beams:

$$
\begin{aligned}
\left\langle\frac{d W}{d t}\right\rangle= & -e E n_{0}\left(\langle u\rangle-\frac{e E_{0}}{m} t\right) \\
& +\frac{1}{2} \varepsilon_{0} E_{1}^{2} \omega_{p}^{2} \int f_{0}(u) d u \frac{d}{d u}\left[u \frac{\sin (\omega t-k u t)}{\omega-k u}\right] \\
& -\varepsilon_{0} E_{1}^{2} \omega_{p}^{2} \frac{e E}{2 m} \int f_{0} d u \frac{d}{d u}\left[\frac{1-\cos (\omega t-k u t)}{(\omega-k u)^{2}}\right] .
\end{aligned}
$$

After a partial integration and using the identities $\lim _{t \rightarrow \infty} \frac{\sin x t}{x}=\pi \delta(x)$ and $\lim _{t \rightarrow \infty} \frac{1-\cos x t}{x}=P\left(\frac{1}{x}\right)$ we finally obtain

$$
\begin{aligned}
\left\langle\frac{d W}{d t}\right\rangle+ & e E n_{0}\left(\langle u\rangle-\frac{e E}{m} t\right) \\
=- & \frac{1}{2} \varepsilon_{0} E_{1}^{2} \omega_{p}^{2} \frac{\pi \omega}{k^{2}} f_{0}^{\prime}\left(\frac{\omega}{k}\right) \\
& +\frac{1}{2} \varepsilon_{0} E_{1}^{2} \frac{e E}{m} \omega_{p}^{2} \int \frac{f_{0}^{\prime}(u)}{(\omega-k u)^{2}} d u .
\end{aligned}
$$

In this expression the left-hand side describes the free motion of plasma particles due to the applied electric field, whereas the right-hand side expresses the different energy transfer processes from the particles to the wave. We emphasize that this energy transfer takes place without collisions and is therefore not connected with an increase of entropy. Consequently, the first term on the right-hand side recovers the known Landau damping.

We examine now the effect of the applied constant electric field $E$, which is described by the second term on the right-hand side of (A6). A long wavelength expansion results in

$$
Q=-\varepsilon_{0} E_{1}^{2} \frac{e E \omega_{p}^{2}}{m \omega^{3}} k+O\left(k^{3}\right),
$$

in agreement with (22), if one multiplies with a factor of 3 to take into account the three dimensions.
[1] R. C. Davidson, in Basic Plasma Physics: Selected Chapters, edited by A. Galeev and R. Sudan (Elsevier Science Publishing B.V., Amsterdam, 1989), Vol. 1, p. 231.

[2] S. Ichimaru, Basic Principles in Plasma Physics (Benjamin, Reading, MA, 1973).

[3] A. B. Michailovskii, Theories of Plasma Instabilities (Consultants Bureau, New York, 1974), Vol. 1.

[4] N. W. Ashcroft and N. D. Mermin, Solid State Physics (Saunders College, Philadelphia, 1976).

[5] D. Lowe and J. R. Barker, J. Phys. C 18, 2507 (1985).

[6] B. Y. Hu and J. W. Wilkins, Phys. Rev. B 39, 8464 (1989).

[7] B. Y. Hu, S. K. Sarker, and J. W. Wilkins, Phys. Rev. B 39, 8468 (1989).

[8] B. Y. Hu and R. F. O'Connell, Phys. Rev. B 40, 3600 (1989).
[9] R. Bertoncini, A. M. Kriman, and D. K. Ferry, Phys. Rev. B 40, 3371 (1989).

[10] R. Bertoncini and A. P. Jauho, Phys. Rev. B 44, 3655 (1991).

[11] R. Bertoncini and A. P. Jauho, Phys. Rev. Lett. 68, 2826 (1992).

[12] K. S. Yi, A. M. Kriman, and D. K. Ferry, Semicond. Sci. Technol. B 7, 316 (1992).

[13] P. Lipavsky, V. Spicka, and B. Velicky, Phys. Rev. B 34, 6933 (1986).

[14] F. S. Khan, J. H. Davies, and J. W. Wilkins, Phys. Rev. B 36, 2578 (1987). Note that we have switched from the canonical momentum $p$ to the kinematical momentum $\mathbf{K}$, as dictated by the requirement of gauge invariance.

[15] A. P. Jauho, in Quantum Transport in Semiconductors, edited by D. Ferry and C. Jacoboni (Plenum Press, New 
York, 1991), Chap. 7.

[16] A. P. Jauho and J. W. Wilkins, Phys. Rev. B 29, 1919 (1984).

[17] K. Morawetz and D. Kremp, Phys. Lett. A 173, 317 (1993).

[18] N. S. Wingreen, C. J. Stanton, and J. W. Wilkins, Phys. Rev. Lett. 57, 1084 (1986).

[19] D. Kremp, K. Morawetz, M. Schlanges, and V. Rietz, Phys. Rev. E 47, 635 (1993).

[20] K. Morawetz, M. Schlanges, and D. Kremp, Phys. Rev. E 48, 2980 (1993).

[21] One should recall that $F(\mathbf{p}, \omega, \mathbf{R}, T)$ is not necessarily a positive semidefinite function, and hence should not be interpreted as a distribution function.

[22] Y. K. Hu, Ph.D. thesis, Cornell University, 1990.

[23] M. Abramowitz and I. A. Stegun, Pocketbook of
Mathematical Functions (Verlag Harri Deutsch, Frankfurt/Main, 1984).

[24] W. D. Kraeft, D. Kremp, W. Ebeling, and G. Röpke, Quantum Statistics of Charged Particle Systems (Akademie Verlag, Berlin, 1986).

[25] E. Lifschitz and L. P. Pitaevsky, in Theoretical Physics, edited by E. Lifschitz (Akademie Verlag, Berlin, 1981).

[26] O. Penrose, Phys. Fluids 3, 258 (1960).

[27] K. Morawetz, Ph.D. thesis, Rostock University, 1992; GSI Report No. GSI-93-14, Darmstadt, 1993 (unpublished).

[28] K. Morawetz and D. Kremp, Phys. Fluids B 1, 225 (1994).

[29] F. F. Chen, Introduction to Plasma Physics and Controlled Fusion (Plenum Press, New York, 1984), Chap. 7, pp. 256-260. 